Radioisotope Distribution Program Progress Report for October 1977

E. Lamb

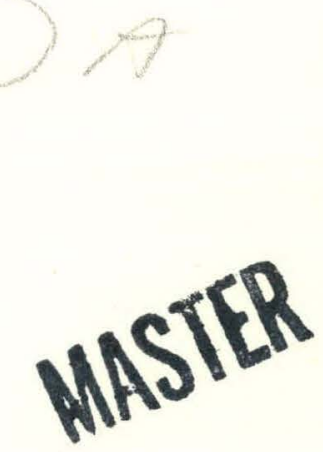

OAK RIDGE NATIONAL LABORATORY OPERATED BY UNION CARBIDE CORPORATION · FOR THE DEPARTMENT OF ENERGY 


\section{DISCLAIMER}

This report was prepared as an account of work sponsored by an agency of the United States Government. Neither the United States Government nor any agency Thereof, nor any of their employees, makes any warranty, express or implied, or assumes any legal liability or responsibility for the accuracy, completeness, or usefulness of any information, apparatus, product, or process disclosed, or represents that its use would not infringe privately owned rights. Reference herein to any specific commercial product, process, or service by trade name, trademark, manufacturer, or otherwise does not necessarily constitute or imply its endorsement, recommendation, or favoring by the United States Government or any agency thereof. The views and opinions of authors expressed herein do not necessarily state or reflect those of the United States Government or any agency thereof. 


\section{DISCLAIMER}

Portions of this document may be illegible in electronic image products. Images are produced from the best available original document. 
This report was prepared as all dicount of work spnnsnred by an agency of the United States Government. Neither the United States Government nor any agency thereof, nor any of their employees, contractors, subcontractors, or their employees, makes any warranty, express or implied, nor assumes any legal liability or responsibility for any third party's use or the results of such use of any information, apparatus, product or process disclosed in this report, nor represents that its use by such third party would not infringe privately owned rights. 


\author{
Contract No. W-7405-eng-26 \\ OPERATIONS DIVISION
}

\title{
RADIOISOTOPE DISTRIBUTION PROGRAM PROGRESS REPORT FOR OCTOBER 1977
}

Date Published - December 1977

E. Lamb

Work Sponsored by

DOE Division of Biomedical and

Environmental Research

NOTICE This document conidius information of a preliminary nature. It is subject to revision or correction and therefore does not represent a final report.

OAK RIDGE NATTONAL LABORATORY

Oak Ridge, Tennessee 37830

operated by

UNION CARBIDE CORPORATION

for the

DEPARTIMENT OF ENERGY 
THIS PAGE

\section{WAS INTENTIONALLY \\ LEFT BLANK}


CONTENTS

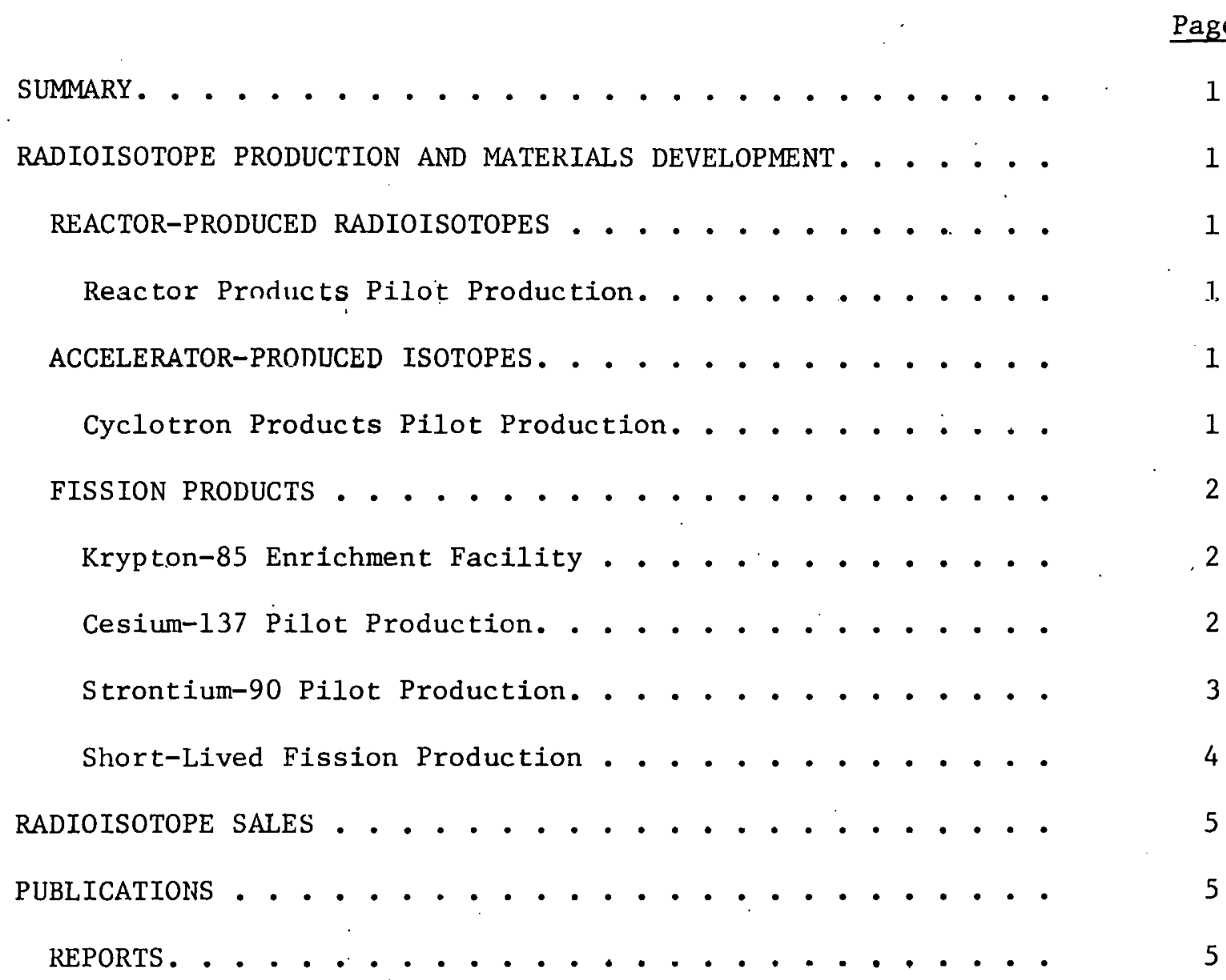


RADIOISOTOPE DISTRIBUTION PROGRAM

PROGRESS REPORT FOR OCTOBER 1977

E. Lamb

SUMMARY

Information is reported on new production, inventory status, operational problems, and radioisotope sales.

RADIOISOTOPE PRODUCTION AND MATERIALS DEVELOPMENT

REACTOR-PRODUCED RADIOISOTOPES

Reactor Products Pilot Production (R. W. Schaich)

(Production and Inventory Accounts)

Processed Units

Radioisotope Amount (mCi)

Calcium-47 25

ACCELERATOR-PRODUCED ISOTOPES

Cyclotron Products Pilot Production (M. R. Skidmore)

(Production and Inventory Accounts)

October 1977 ORNL 86-Inch Cyclotron runs for ORNL and non-ORNL programs are given in Table 1 .

Table 1. Cyclotron Irradiations and Runs for October 1977

\begin{tabular}{|c|c|c|c|c|c|}
\hline Date & Customer & Product & Target & $\begin{array}{c}\text { Total Time } \\
\text { (hr:min) }\end{array}$ & $\begin{array}{c}\text { Total } \\
\text { Charges } \\
\end{array}$ \\
\hline & $\underline{\mathrm{OF}}$ & RNL Programs & & & \\
\hline \multirow[t]{2}{*}{$\begin{array}{l}10-6-77 \\
10-27-77\end{array}$} & $\begin{array}{l}\text { ORAU } \\
\text { ORAU }\end{array}$ & $\begin{array}{l}\text { Carbon-11 } \\
\text { Carbon-11 }\end{array}$ & $\begin{array}{l}\text { Boron Oxide } \\
\text { Boron Oxide }\end{array}$ & $\begin{array}{l}6: 20 \\
5: 35 \\
\end{array}$ & $\begin{array}{l}755 \\
668 \\
\end{array}$ \\
\hline & & & & $11: 55$ & $\$ 1,423$ \\
\hline \multicolumn{6}{|c|}{-1} \\
\hline $\begin{array}{l}10-3-77 \\
10-7-77 \\
10-11-77 \\
10-18-77 \\
10-21-77 \\
10-25-77 \\
10-28-77\end{array}$ & $\begin{array}{l}\text { New England Nuclear } \\
\text { New England Nuclear } \\
\text { Westinghouse-Hanford } \\
\text { New England Nuclear } \\
\text { New England Nuclear } \\
\text { New England Nuclear } \\
\text { New England Nuclear }\end{array}$ & $\begin{array}{l}\text { Gallium-67 } \\
\text { Guld=195 } \\
\text { Beryllium-7 } \\
\text { Zinc-65 } \\
\text { Germanium-68 } \\
\text { Gallium-67 } \\
\text { Germanium-68 }\end{array}$ & $\begin{array}{l}\text { Zinc-68 } \\
\text { Platinum } \\
\text { Lithium } \\
\text { Copper } \\
\text { Gallium } \\
\text { Zinc-68 } \\
\text { Gallium }\end{array}$ & $\begin{array}{r}42: 25 \\
11: 15 \\
2: 15 \\
6: 15 \\
13: 15 \\
25: 15 \\
13: 15 \\
\end{array}$ & $\begin{array}{r}6,555 \\
1,918 \\
670 \\
1,130 \\
2,279 \\
3,980 \\
2,279 \\
\end{array}$ \\
\hline $10-28-77$ & & & & $113: 55$ & $\$ 18,811$ \\
\hline \multicolumn{6}{|c|}{ Isotopes Sales Inventory } \\
\hline $10-13-77$ & Isotopes Sales & Cobalt -57 & Nickel & $49: 30$ & $\$ 8,207$ \\
\hline
\end{tabular}




\section{Cyclotron Operations}

Other than the routine maintenance of the cyclotron, the only unusual event during the month was a power outage October 1, 1977. Firemen checking the fire alarm system in the building tripped the 13,800 volt transformer supplying the building, resulting in the loss of vacuum in the cyclotron tank and the usual accompanying complications.

\section{FISSION PRODUCTS}

Krypton-85 Enrichment Facility (R. W. Schaich)

'I'wo of the ${ }^{85} \mathrm{Kr}$ enrichment columns were operative during the month of October and all the units were functioning according to design. $\Lambda l l$ three columns in the south bank. were unloaded and the yield ranged from 5 to $21.84 \%$ enrichment in ${ }^{85} \mathrm{Kr}$. A total of $175 \mathrm{Ci}$ of $21.84 \%$ enriched ${ }^{85} \mathrm{Kr}$ was made available for sales. The remaining cuts varied from 5 to $10 \%$ and will be reloaded on the south bank columns in November. The south bank loading station was modified to increase the operating sensitivity and to reduce personnel exposure from the higher enrichment $85 \mathrm{Kr}$ being handled in the system.

Cesiuri-137 Pilot Production (R. W. Schaich) (Production and Inventory Accounts)

1. Process Status

The ${ }^{137} \mathrm{Cs}$ processing equipment has been placed in standby status.

2. Operational Summary

\section{Product Inventory}

(Decay calculated through August 31, 1977)

Inventory Material

Cesium-137 chloride powder

Tuldl Invenicury Maceriā
Amount (Ci)

$\underline{29,960}$

29,960 
Non-Inventory Material

Special Form Cans

Material returned or stored for customer Nuclear Research Corporation

J. L. Shepherd

- New England Nuclear Corporation

Puerto Rico Sources

Lockheed

AECL powder

Radiation Resources

Minn. Mining \& Mfg. Company

Gamma Industries

Total Non-Inventory Material

TOTAL INVENTORY AND NON-INVENTORY MATERIAL
Amount (Ci)

$$
\begin{array}{r}
4,300 \\
0 \\
50,600 \\
2,700 \\
7,900 \\
19,600 \\
71,500 \\
19,800 \\
2,800 \\
8,400 \\
\hline 187,600 \\
\hline
\end{array}
$$

217,560

Fabrication Summary

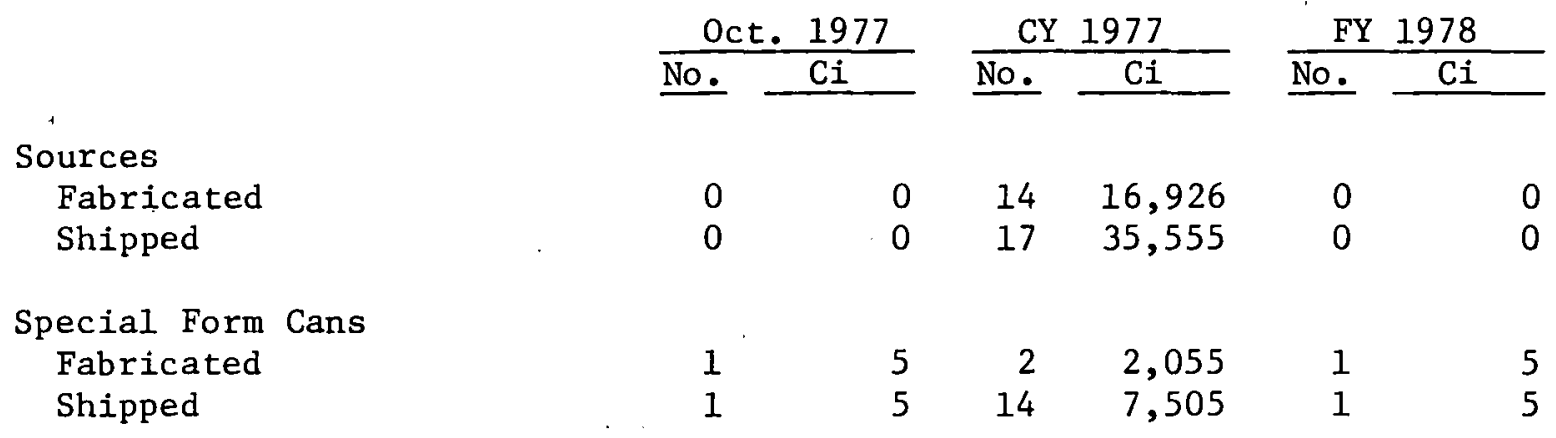

3. Current Orders

All orders on hand have been completed and the material placed into storage awaiting receipt of release for the material.

Strontium-90 Pilot Production ( $R$. W. Schaich)

(Production and Inventory Accounts)

1. Process Status

The 90 source fabrication equipment has been placed in standby status. 
Product Inventory

(Decay calculated through August 31, 1977)

Inventory Material

Amount (Ci)

${ }^{90} \mathrm{Sr}$ titanate powder $( \pm 5 \%)$

Sources in fabrication

Stock powder cans

Stock solution

0$$
3,325
$$

200

3,525

Non-Inventory Materiàl

Batsh 2.6Sr-74RE

Calorimeter Standards

Weacher Bureau source

SNAP-7B

SNAP-7C

SNAP-7D

SNAP material purchase ${ }^{a}$

AGN-4 Powder

Total Non-Inventory Material

TOTAL INVENTORY AND NON-INVENTORY MATERIAL
Amount (Ci)

$$
7,900
$$$$
4,800
$$

11,400

$1.56,300$

24,600

143,000

248,300

38,400

634,700

638,225

${ }^{a}$ Strontium-90 purchased under DRRD program.

Fabrication Summary

\begin{tabular}{|c|c|c|c|c|c|c|}
\hline & & 1977 & & 1977 & & 978 \\
\hline & No. & $\mathrm{Ci}$ & No. & $\mathrm{Ci}$ & No. & $C 1$ \\
\hline Sources & & & & & & \\
\hline Fabricated & 0 & 0 & 3 & 157,000 & 0 & 0 \\
\hline Shipped & 0 & 0 & 4 & 177,000 & 0 & 0 \\
\hline Sperial Form & & & & & & \\
\hline Fabricated & 0 & 0 & 0 & 0 & 0 & 0 \\
\hline Shipped & 0 & 0 & 2 & 20 & 0 & 0 \\
\hline
\end{tabular}

Short-Lived Fission Production (n. W. Sohaich)

(Production and Inventory Accounts)

\section{Isotope}

Xenon-133

Niobium-95

Iodine-131
Number of Batches

2

1

1
Amount (Ci)

1500

13 


\title{
RADIOISOTOPE SALES
}

\author{
J. E. Ratledge
}

Shipments made during the month that may be of interest are listed below:

Customer

Isotope

Amount

\section{Large Quantities}

U. S. Radium Corporation

New England Nuclear Corporation

American Atomics Corporation

TCN Pharmaceuticals

Self-Powered Lighting, Ltd.

Schwarz/Mann

\begin{abstract}
Tritium
Tritium

Tritium

Tritium

Tritium

Tritium
\end{abstract}

$10,000 \mathrm{Ci}$
$8,000 \mathrm{Ci}$
$45,000 \mathrm{Ci}$
$1,000 \mathrm{Ci}$
$3,000 \mathrm{Ci}$
$1,500 \mathrm{Ci}$

Items Used in Cooperative Programs
University of Kentucky

University of Southern California
Platinum-195m

Platinum-195m
$20 \mathrm{mCi}$

$20 \mathrm{mCi}$

The radioisotope sales and shipments for October 1976 and the first month of fiscal year 1978 are given in Table 2 .

Table 2. Radioisotope Sales and Shipments

\begin{tabular}{|c|c|c|c|c|}
\hline Item & \multicolumn{2}{|c|}{$\begin{array}{l}10-1-76 \text { thru } \\
10-31-76\end{array}$} & \multicolumn{2}{|c|}{$\begin{array}{l}10-1-77 \text { thru } \\
10-31-77\end{array}$} \\
\hline $\begin{array}{l}\text { Inventory items } \\
\text { Major products } \\
\text { Radioisotope services } \\
\text { Cyclotron irradiations } \\
\text { Miscellaneous processed materials } \\
\text { Packing and Shipping }\end{array}$ & $\$$ & $\begin{array}{r}26,165 \\
7,378 \\
7,504 \\
44,573 \\
9,571 \\
13,990 \\
\end{array}$ & $\$$ & $\begin{array}{r}88,070 \\
11,051 \\
40,118 \\
24,688 \\
4,909 \\
15,415 \\
\end{array}$ \\
\hline Total & $\$$ & 109,181 & $\$$ & 184,251 \\
\hline Number of shipments & & 178 & & 186 \\
\hline
\end{tabular}

\section{PUBLICATIONS}

REPORTS

E. Lamb, Radioisotope Distribution Progrom Progress Report for September 1977, ORNL/TM-6154, Oak Ridge National Laboratory (November 1977). 
. i

THIS PAGE

WAS INTENTIONALLY

LEFT BLANK 
1. F. N. Case

2. W. R. Casto

3. J. A. Cox

4. R. F. Hibbs

5. E. Lamb

6. H. H. Nichol

7. C. L. Ottinger

8. J. K. Poggenburg

9. H. Postma
10. M. E. Ramsey

11. J. E. Ratledge

12. C. R. Richmond

13. R. W. Schaich

14. M. R. Skidmore

15. M. J. Skinner

16-17. Central Research Library

18-19. Laboratory Records Department

20. Laboratory Records - RC

21. Document Reference Section

EXTERNAL DISTRIBUTTON

22. B. J. Dropesky, LASL, Los Alamos, New Mexico

23-24. J. H. Jarrett, PNL, Kichland Washington

25. D. K. Jones, Richland Operations office, Richland, Washington

26. J. W. Maddox, DOE-DBER, Washington, D.C.

27. H. A. O'Brien, LASL, Los Alamos, New Mexico

28. F. J. Skozen (Krizek), Argonne Cancer Research Hospital, Chicago

29. L. G. Stang, Jr., BNL, New York

30. W. H. Weyzen, DOE-DBER, Washington, D.C.

31-32. R. W. Wood, DOE-DBER, Washington, D.C.

33. Donner Laboratory Library, Univ. of California, Berkeley, Calif., 94720

34. Research and Technical Support Division, ORO

35-36. Technical Information Center 